\title{
Autoreversibility: exploiting symmetries in Markov chains
}

\author{
Andrea Marin \\ DAIS - Università Ca' Foscari Venezia \\ Venezia, Italy \\ Email: marin@dais.unive.it
}

\author{
Sabina Rossi \\ DAIS - Università Ca’ Foscari Venezia \\ Venezia, Italy \\ Email: srossi@dais.unive.it
}

\begin{abstract}
The computation of the steady-state distribution of Continuous Time Markov Chains (CTMCs) may be a computationally hard problem when the number of states is very large. In order to overcome this problem, in the literature, several solutions have been proposed such as the reduction of the state space cardinality by lumping, the factorisation based on productform analysis and the application of the notion of reversibility. In this paper we address this problem by introducing the notion of autoreversibility which is defined as a symmetric coinductive relation which induces an equivalence relation among the chain's states. We show that all the states belonging to the same equivalence class share the same stationary probabilities and hence the computation of the the steady-state distribution can be computationally more efficient. The definition of autoreversibility takes inspiration by the Kolmogorov's criteria for reversible processes and hence requires to test a property on all the minimal cycles of the chain. We show that the notion of autoreversibility is different from that of reversible processes and does not correspond to other state aggregation techniques such as lumping. Finally, we discuss the applicability of our results in the case of models defined in terms of a Markovian process Algebra such as the Performance Evaluation Process Algebra.
\end{abstract}

\section{INTRODUCTION}

Discrete and continuous time Markov Chains are the theoretical foundations of many approaches to the performance evaluation of computer systems. In particular, many formalisms that have been introduced for defining performance and reliability models have an underlying stochastic process that is a Markov chain. To mention some examples, queueing networks may be defined both with discrete or continuous time (see, [1] for a recent survey), while stochastic Petri nets [2], Markovian process algebras [3], [4] and stochastic automata networks [5] have underlying Continuous Time Markov Chains (CTMCs). The analysis of the underlying process allows one to derive the desired performance indices of the model. Often these indices are computed in steady-state (if it exists), i.e., we compute the stationary state probabilities when the time elapsed from the initial instant tends to infinity. A discrete or continuous time Markov chain that is ergodic admits a unique limiting distribution that is called steady-state distribution. The conditions for ergodicity are well-known for both the discrete and continuous time cases (see, e.g., [6]).

Hereafter, we focus on CTMCs but the results we are presenting may be straightforwardly reformulated for the discrete time case. A CTMC is characterised by its infinitesimal generator $\mathbf{Q}=\left(q_{i j}\right)$, i.e., a square matrix whose dimension corresponds to the number of model's states, and each entry $q_{i j}$, for $i \neq j$, is the transition rate from state $i$ to state $j$, while $q_{i i}$ is the opposite of the sum of the row extra-diagonal entries. Let $\boldsymbol{\pi}=\left(\pi_{i}\right)$ be the row vector associating each state $i$ with its steady-state probability $\pi_{i}, 0<\pi_{i} \leq 1$. Then, $\pi$ is the unique vector that satisfies the following matrix equations:

$$
\boldsymbol{\pi} \mathbf{Q}=\mathbf{0} \wedge \boldsymbol{\pi} \mathbf{1}^{T}=1,
$$

where 1 is the row vector of 1 with the right dimension. The first system of equations is usually referred to as the system of global balance equations (GBEs) while the second equation is the normalising condition that ensures that the sum of the probabilities is 1 since for ergodic chains the rank of $\mathbf{Q}$ is $n-1$, where $n$ is the number of states. In practice, for large chains, solving the GBEs can be a computationally difficult task since the algorithms may turn to be numerically unstable and time consuming. In the literature, several methods have been introduced to deal with this problem. Exact and approximate solutions of the GBEs are discussed in [7], [6]. A different approach is that taken by the product-form theory that allows for the derivation of the steady-state distribution as normalised product of the steady-state distributions of the model's sub-components considered in isolation and opportunely parametrised [8], [9], [10], [11], [12], [13]. Neuts and other authors proposed a way to exploit the block-regular structure of a certain class of infinitesimal generators [14]. The approach we propose here aims at avoiding the solution of the GBE system of a CTMC by exploiting some structural properties that also leads to a reduction of the number of states that must be considered. Although the idea of reducing the number of states of a Markov process is the base of the aggregation and the lumping techniques studied for instance in [15], to the best of our knowledge, the results we propose are novel. Specifically, we introduce the notion of autoreversibility for CTMCs which relies on a symmetric coinductive relation, named reversal bisimulation, which induces an equivalence relation among the chain's states called reversal equivalence. The formal definition of this equivalence relation is inspired by the generalised Kolmogorov's criteria proved in [9]. However, while in [9] and in [16] the authors deal with two distinct processes, the original CTMC and its reversed, in this paper 
we study only the forward process and use similar criteria to identify some internal symmetries that allow us to simplify the system of GBE. A CTMC is autoreversible if every state $i$ is associated with another state $i^{\prime}$ that could be interpreted as its reversed. Two states belong to the same equivalence class if they share the same reversed states. We prove that all the states belonging to the same equivalence class must have the same steady-state probability and that given the stationary probability of state $i$, we can easily compute that of $i^{\prime}$ which depends also on the cardinality of the equivalence classes of $i$ and $i^{\prime}$. These properties allow the definition of extremely efficient ways for the computation of the steadystate distribution.

The paper is structured as follows. Section II discusses some related work and introduces the notation and the basic notions needed to keep the paper self-contained. Section III introduces the notion of weak similarity among states which is at the basis of the definition of autoreversibility. Section IV introduces the definition of autoreversibility and discusses the properties of autoreversible CTMCs. Section V shows the application of these results for models specified using the Markovian process algebra PEPA. Section VI concludes the paper.

\section{THEORETICAL BACKGROUND}

In this section we introduce the notion of reversibility of Markov chains with the aim of helping the intuition behind the definition of autoreversibility and reviewing the existing literature. For the sake of brevity we just consider CTMCs although the theory of reversibility can be formulated also for Discrete Time Markov Chains.

An ergodic CTMC with state space $S$ admits a unique steady-state distribution. Let $\mathbf{Q}=\left(q_{i j}\right)$ be its infinitesimal generator, then the steady-state distribution $\pi$ is the unique vector that satisfies the equation:

$$
\boldsymbol{\pi} \mathbf{Q}=\mathbf{0} \wedge \sum_{s \in S} \pi_{s}=1 .
$$

Any non-trivial solution of the GBE differs by a constant (invariant measure) but only one satisfies the normalising condition.

Given an ergodic CTMC in steady-state $X(t)$ with $t \in$ $\mathbb{R}^{+}$, we call $X(\tau-t)$ its reversed process. It can be shown that $X(\tau-t)$ is also a stationary CTMC. We say that $X(t)$ is reversible if it is stochastically identical to $X(\tau-t)$, i.e., $X_{t_{1}}, \ldots, X_{t_{n}}$ has the same distribution of $\left(X_{\tau-t_{1}}, \ldots, X_{\tau-t_{n}}\right)$ for all $t_{i}, \tau \in \mathbb{R}^{+}[16$, Ch. 1].

A CTMC is reversible if and only if the following detailed balance equations are satisfied [16, Theorem 1.3]:

$$
\pi_{i} q_{i j}=\pi_{j} q_{j i} \text { for all states } i \neq j,
$$

where $\mathbf{Q}=\left(q_{i j}\right)$ is the process's instantaneous state transition rate matrix (its generators, apart from the diagonal) and $\pi$ is the vector associated with its steady-state distribution. Clearly, a reversible CTMC $X(t)$ and its dual $X(\tau-t)$ have the same steady-state distribution.
An important property of reversible CTMCs is the Kolmogorov's criterion which states that the reversibility of a process can be established directly from its transition rates.

Proposition 1 (Kolmogorov's criteria [16]). A stationary Markov process with state space $S$ and infinitesimal generator $\mathbf{Q}$ is reversible if and only if its transition rates satisfy the following equation: for every finite sequence of states $i_{1}, i_{2}, \ldots i_{n} \in S$.

$$
q_{i_{1} i_{2}} q_{i_{2} i_{3}} \cdots q_{i_{n-1} i_{n}} q_{i_{n} i_{1}}=q_{i_{1} i_{n}} q_{i_{n} i_{n-1}} \cdots q_{i_{3} i_{2}} q_{i_{2} i_{1}} .
$$

Notice that the reversed process $X(\tau-t)$ can still be defined when $X(t)$ is not reversible. In [9] the author shows that $X(\tau-t)$ is a CTMC and proves that the transition rates are defined in terms of the stationary distribution of the process $X(t)$ as stated by the following proposition.

Proposition 2 (Reversed process transition rates [9]). Given the stationary Markov chain $X(t)$, the transition rates of the reversed process $X(\tau-t)$ are defined as follows:

$$
q_{j i}^{R}=\frac{\pi_{i}}{\pi_{j}} q_{i j},
$$

where $q_{j i}^{R}$ denotes the transition rate from state $j$ to $i$ in the reversed process. It still holds that the forward and the reversed process have the same stationary distribution.

Observe that we can replace in Equation (2) any nontrivial solution of the GBE. Roughly speaking, we can say that the knowledge of the reversed process' transition rates allows for an efficient computation of the invariant measure of the process and vice versa the latter allows for an efficient definition of the reversed process. In [9] the author generalises the Kolmogorov's criteria in order to encompass non-reversible CTMCs. Hereafter, for a given state $i$ we denote by $q_{i}$ (resp. $q_{i}^{R}$ ) the quantity $\sum_{j \in S, i \neq j} q_{i j}$ (resp. $\sum_{j \in S, i \neq j} q_{i j}^{R}$ ).

Proposition 3 (Kolmogorov's generalised criteria [9]). A stationary Markov process with state space $S$ and infinitesimal generator $\mathbf{Q}$ has reversed process with infinitesimal generator $\mathbf{Q}^{R}$ if and only if the following conditions hold:

1) $q_{i}^{R}=q_{i}$ for every state $i \in S$;

2) for every finite sequence of states $i_{1}, i_{2}, \ldots, i_{n} \in S$,

$$
q_{i_{1} i_{2}} q_{i_{2} i_{3}} \ldots q_{i_{n 1} i_{n}} q_{i_{n} i_{1}}=q_{i_{1} i_{n}}^{R} q_{i_{n} i_{n-1}}^{R} q_{i_{2} i_{3}}^{R} q_{i_{2} i_{1}}^{R} .
$$

Proposition 3 suggests us a proof method for verifying whether a vector $\boldsymbol{\pi}$ satisfies the GBE system $\boldsymbol{\pi Q}=\mathbf{0}$ for a given process $X(t)$. It consists in:

1) defining the reversed process $X(\tau-t)$ using Proposition 2 and assuming $\pi$,

2) verifying the generalised Kolmogorov's criteria of Proposition 3.

If the generalised Kolmogorov's criteria are verified and $\sum_{s \in S} \pi_{s}=1$ then, by uniqueness of the steady-state distribution, we can conclude that $\pi$ is the steady-state distribution of the process.

The last notion we introduce in this section is that of similar states proposed by Yap in [17]. 
Definition 1 (Similar states). Two distinct states $i_{1}$ and $i_{2}$ of a CTMC are similar if their rates to every other state agree, i.e.:

$$
\forall j \neq i_{1}, i_{2} \quad q_{i_{1} j}=q_{i_{2} j}
$$

In [17] the author proves that the similarity relation is not transitive (and hence it is not an equivalence relation). Moreover, given a partition of the chain's state space into similarity classes ${ }^{1} S_{1}, \ldots, S_{t}$ then $S_{1}, \ldots, S_{t}$ is a lumping [15] for the original CTMC. In [17] the author discusses the applicability of this result with special attention to the analysis of DNA sequences.

\section{WEAK SIMILARITY OF STATES}

In this section we generalize the Yap's notion of similarity among states by relating those states whose rates to (and from) any class of weakly similar states agree. We show that weak similarity is an equivalence relation.

In the following, $i \rightarrow j$ denotes a transition from state $i$ to state $j$ and $[j]_{\sim_{w}}$ denotes the set of states which are weakly similar to $j$. Moreover, we denote by $m_{i[j]_{\sim}}$ the number of transitions from state $i$ to the set of states $[j]_{\sim_{w}}$ and by $m_{[j]_{\sim_{w}} i}$ the number of transitions from the set $[j]_{\sim_{w}}$ to $i$.

Definition 2 (Weak similarity). Given a CTMC with state space $S$, a relation $\sim_{w} \subseteq S \times S$ is a weak similarity if:

1) For every $i_{1} \rightarrow j_{1}$ and $i_{2} \rightarrow j_{2}$ such that $i_{1} \sim_{w} i_{2}$ and $j_{1} \sim_{w} j_{2}$ it holds that

$$
q_{i_{1} j_{1}}=q_{i_{2} j_{2}}
$$

2) for every state $j$ and for every state $i_{1}$ and $i_{2}$ such that $i_{1} \sim_{w} i_{2}$, it holds that:

$$
\begin{aligned}
& m_{i_{1}[j]_{\sim_{w}}}=m_{\left.i_{2}[j]\right]_{w}} \\
& m_{[j]_{\sim_{w}} i_{1}}=m_{[j]_{\sim_{w}} i_{2}}
\end{aligned}
$$

where for every $i, j \in S, m_{i[j] \sim_{w}}=\left|\left\{i \rightarrow k: j \sim_{w} k\right\}\right|$ and $m_{[j]_{\sim_{w}} i}=\left|\left\{k \rightarrow i: j \sim_{w} k\right\}\right|$.

Before giving the intuition behind each of the definition items, we prove the following proposition.

Proposition 4. The weak similarity is an equivalence relation.

Proof: Reflexivity and symmetry follow immediately. We prove that $\sim_{w}$ is transitive. Let $i_{1}, i_{2}, i_{3}$ be three states such that $i_{1} \sim_{w} i_{2}$ and $i_{2} \sim_{w} i_{3}$.

In order to prove the first item of Definition 2 , let $j_{1}, j_{2}, j_{3}$ be three states such that $i_{1} \rightarrow j_{1}, i_{2} \rightarrow j_{2}$ and $i_{3} \rightarrow j_{3}$ and $j_{1} \sim_{w} j_{2}$ and $j_{2} \sim_{w} j_{3}$. From $i_{1} \sim_{w} i_{2}$ we have $q_{i_{1} j_{1}}=q_{i_{2} j_{2}}$ and from $i_{2} \sim_{w} i_{3}$ we have $q_{i_{2} j_{2}}=q_{i_{3} j_{3}}$, hence $q_{i_{1} j_{1}}=q_{i_{3} j_{3}}$.

To prove the second item of Definition 2 consider a state $j$. From $i_{1} \sim_{w} i_{2}$ we have $m_{i_{1}[j]_{\sim_{w}}}=m_{i_{2}[j]_{\sim_{w}}}$ and $m_{[j]_{\sim_{w}} i_{1}}=$ $m_{[j]_{\sim_{w}} i_{2}}$. From $i_{2} \sim_{w} \quad i_{3}$ we have $m_{i_{2}[j]_{\sim_{w}}}=m_{i_{3}[j]_{\sim_{w}}}$ and $m_{[j]_{\sim_{w}} i_{2}}=m_{[j]_{\sim_{w}} i_{3}}$. Hence $m_{i_{1}[j]_{\sim_{w}}}=m_{i_{3}[j]_{\sim_{w}}}$ and $m_{[j]_{\sim_{w}} i_{1}}=m_{[j]_{\sim_{w}} i_{3}}$.

Informally, Condition 1 of Definition 2 asks that all the transitions from the states of an equivalence class $[i]_{\sim_{w}}$ to

\footnotetext{
${ }^{1}$ Note that two elements belonging to the same class are similar but two similar elements can belong to different classes.
}

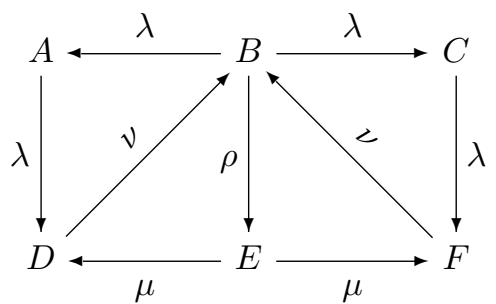

Fig. 1: CTMC studied in Example 2 (running example).

any state of an equivalence class $[j]_{\sim_{w}}$ have the same rate. Condition 2 asks that the number transitions from equivalent states to the states of a fixed equivalence class are the same, and the number transitions from the states of a fixed equivalence class to distinct equivalent states are the same.

Notice that Yap's notion of similarity classes does not always imply weak similarity. Indeed, let us consider the example presented in [17] of a CTMC with infinitesimal generator

$$
\mathbf{Q}=\left[\begin{array}{ccc}
* & \alpha_{2} & \alpha_{3} \\
\alpha_{1} & * & \alpha_{3} \\
\alpha_{1} & \alpha_{4} & *
\end{array}\right]
$$

where $*$ denotes the opposite of the sum of the row extra diagonal entries and all $\alpha_{i}$ are distinct. Notice that according to Definition 1 state 1 is similar to state 2 and the latter is similar to state 3 . However, states 1 and 3 are not similar since $\alpha_{2} \neq$ $\alpha_{4}$. Notice that in the case of weak similarity if $1 \sim_{w} 2$, then it must hold $\alpha_{1}=\alpha_{2}$ by Condition 1 of Definition 2. In [17] the author proves that for reversible CTMC, state similarity becomes an equivalence relation. In these cases it implies our notion of weak similarity.

The following example shows a CTMC and its partition into equivalence classes according to $\sim_{w}$.

Example 1 (Weak similarity). Let us consider the CTMC depicted by Figure 1.

We can easily prove that $A \sim_{w} C, D \sim_{w} F$ and hence the state space $S$ is partitioned as follows:

$$
S / \sim_{w}=\{\{A, C\},\{D, F\},\{B\},\{E\}\} .
$$

The following Propositions 5 and 6 and Theorem 1 allow us to characterise the steady-state distribution of an ergodic CTMC on which a weak similarity relation has been defined.

Proposition 5. Given a CTMC with state space $S$, if $i, j \in S$ and $i \sim \sim_{w} j$ then $q_{i}=q_{j}$ where $q_{h}=\sum_{k \in S \backslash\{h\}} q_{h k}$ is the total rate out of state $h$, with $h=i, j$.

Proof: The proof immediately follows by Definition 2 .

Proposition 6. Given a CTMC with state space $S$ and a weak similarity relation $\sim_{w} \subseteq S \times S$, then $S / \sim_{w}$ is a strong lumping of the chain.

Proof: Consider the states $i_{1}, i_{2} \in S$ and let $i_{1} \sim_{w} i_{2}$. We must prove that taken any other equivalence class $[j]_{\sim_{w}}$ 
the following relation holds [15]:

$$
\sum_{j \in[j]_{\sim_{w}}} q_{i_{1} j}=\sum_{j \in[j]_{\sim_{w}}} q_{i_{2} j}
$$

This immediately follows by Definition 2 .

Another interesting property of weak similarity that will be used in Section IV is the following:

Proposition 7. Given a CTMC and a weak similarity relation $\sim_{w} \subseteq S \times S$, for each class $[i]_{\sim_{w}},[j]_{\sim_{w}} \in S / \sim_{w}$ it holds:

$$
n_{i} m_{i[j]_{\sim_{w}}}=n_{j} m_{[i]_{\sim_{w}} j}
$$

where $n_{h}$ is the cardinality of equivalence class $[h]_{\sim_{w}}$, with $h=i, j$.

Proof: The proof follows from Definition 2 by observing that the total number of arcs from class $[i]_{\sim_{w}}$ to $[j]_{\sim_{w}}$ can be computed using either the expression on the left-hand-side or that on the right-hand-side of Equation (4).

Theorem 1. Given an ergodic CTMC with state space $S$ and a weak similarity relation $\sim_{w} \subseteq S \times S$ and two states $i_{1}, i_{2} \in S$, such that $i_{1} \sim_{w} i_{2}$, then $\pi_{i_{1}}=\pi_{i_{2}}$.

Proof: Since $\sim_{w}$ induces a lumping of the CTMC we can write down the system of Global Balance Equations (GBEs) of the lumped process:

$$
\pi_{[i]_{\sim_{w}}} \sum_{[j]_{\sim_{w}}} q_{i j} m_{i[j]_{\sim_{w}}}=\sum_{[j]_{\sim_{w}}} \pi_{[j]_{\sim_{w}}} q_{j i} m_{j[i]_{\sim_{w}}},
$$

where $\pi_{[h]_{\sim_{w}}}=\sum_{h \in[h]_{\sim_{w}}} \pi_{h}$, for $h=i, j$. We now prove that $i_{1} \sim_{w} i_{2}$ implies $\pi_{i_{1}}=\pi_{i_{2}}$ by substitution in the system of GBE. So let us assume the steady-state distribution $\pi_{h}=\pi_{[h]_{\sim_{w}}} / n_{h}$, where $n_{h}$ denotes the cardinality of $[h]_{\sim_{w}}$. Therefore, the GBE associated with state $i$ is:

$$
\frac{\pi_{[i]_{\sim_{w}}}}{n_{i}} \sum_{j} q_{i j}=\sum_{j} \frac{\pi_{[j]_{\sim_{w}}}}{n_{j}} q_{j i},
$$

which can be rewritten as:

$$
\frac{\pi_{[i]_{\sim_{w}}}}{n_{i}} \sum_{[j]_{\sim_{w}}} \sum_{j \in[j]_{w}} q_{i j}=\sum_{[j]_{\sim_{w}}} \frac{\pi_{[j]_{\sim_{w}}}}{n_{j}} \sum_{j \in[j]_{\sim_{w}}} q_{j i},
$$

where we notice that $\sum_{j \in[j]_{w}} q_{i j}=m_{i[j]_{\sim_{w}}} q_{i j}$ and $\sum_{j \in[j]_{\sim_{w}}} q_{j i}=m_{[j]_{\sim_{w}}} q_{j i}$ where $q_{i j}$ and $q_{j i}$ which appears on the right-hand-side of these equalities represent the positive rate of an arbitrary transition between a sate of classes $i$ and $j$ or $j$ and $i$, respectively (this is sound by Definition 2). Therefore, we have:

$$
\frac{\pi_{[i]_{\sim_{w}}}}{n_{i}} \sum_{[j]_{\sim_{w}}} m_{i[j]_{\sim_{w}}} q_{i j}=\sum_{[j]_{\sim_{w}}} \frac{\pi_{[j]_{\sim_{w}}}}{n_{j}} m_{[j]_{\sim_{w}}} q_{j i} .
$$

We can multiply both hand-sides by $n_{i}$ and observe that by Proposition 7 we have $m_{[j]_{\sim_{w}} i} / n_{j}=m_{j[i]_{\sim_{w}}} / n_{i}$ we obtain again Equation (5) which is an identity.

\section{AutoreVERsibility}

In this section we introduce the notion of autoreversibility for a given Markov process through the definition of two relations over its states: a reversal bisimulation which allows us to relate "reversed" sates and a reversal equivalence relation which relates states corresponding to the same class of reversed states. We also study the properties of autoreversible CTMCs and, in particular, the relationships among the stationary probabilities of its states. Henceforth we will consider only stationary Markov chains.

The reversal bisimulation over the states of CTMC is a coinductive definition, in the style of bisimulation [18], formally expressed ad follows.

Definition 3 (Reversal bisimulation). Consider an ergodic CTMC with state space $S$ and infinitesimal generator Q. A symmetric relation $\mathcal{R} \subseteq S \times S$ is a reversal bisimulation if

1) for every $\left(i, i^{\prime}\right) \in \mathcal{R}, q_{i}=q_{i^{\prime}}$;

2) for every $\left(i_{1}, i_{1}^{\prime}\right) \in \mathcal{R}$ and for every finite sequence of one-step transitions $i_{1} \rightarrow i_{2} \rightarrow \ldots \rightarrow i_{n-1} \rightarrow i_{n}$ there exist $i_{n}^{\prime} \rightarrow i_{n-1}^{\prime} \rightarrow \cdots \rightarrow i_{2}^{\prime} \rightarrow i_{1}^{\prime}$ such that $\left(i_{k}, i_{k}^{\prime}\right) \in$ $\mathcal{R}$ for all $k \in\{1, \ldots, n\}$ and

$$
q_{i_{1} i_{2}} q_{i_{2} i_{3}} \cdots q_{i_{n-1} i_{n}} q_{i_{n} i_{1}}=q_{i_{1}^{\prime} i_{n}^{\prime}} q_{i_{n}^{\prime} i_{n-1}^{\prime}} \cdots q_{i_{3}^{\prime} i_{2}^{\prime}} q_{i_{2}^{\prime} i_{1}^{\prime}} .
$$

We are interested in the relation which is the largest reversal bisimulation, formed by the union of all reversal bisimulations.

The following proposition ensures that any union of reversal bisimulations is itself a reversal bisimulation.

Proposition 8. Consider an ergodic CTMC with state space $S$ and infinitesimal generator $\mathbf{Q}$. Let $\mathcal{R}_{1}, \mathcal{R}_{2} \subseteq S \times S$ be two reversal bisimulations. Then $\mathcal{R}_{1} \cup \mathcal{R}_{2}$ is a reversal bisimulation.

Proof: Let $\left(i, i^{\prime}\right) \in \mathcal{R}_{1} \cup \mathcal{R}_{2}$. Then either $\left(i, i^{\prime}\right) \in \mathcal{R}_{1}$ or $\left(i, i^{\prime}\right) \in \mathcal{R}_{2}$ and hence Conditions 1 and 2 of Definition 3 are satisfied.

Based on the above result we can define the maximal reversal bisimulation as the union of all reversal bisimulations.

Definition 4 (Reversal bisimilarity). Given an ergodic CTMC with state space $S$, we denote by $\sim_{r}$ the maximal reversal bisimulation over $S$ which is defined by

$$
\sim_{r}=\bigcup\{\mathcal{R} \mid \mathcal{R} \text { is a reversal bisimulation }\} .
$$

If $\sim_{r} \subseteq S \times S$ is complete, i.e., for all $i \in S$ there exists $i^{\prime} \in S$ such that $i \sim_{r} i^{\prime}$, then $\sim_{r}$ is called reversal bisimilarity over $S$.

It is worth notice that the effective computation of reversal bisimilarity over a finite state space chain can be implemented as a reformulation of the well-known algorithms that have been developped in the literature of formal models for bisimulation [19], [20].

Notice that reversal bisimilarity $\sim_{r}$ is symmetric but in general it is neither reflexive nor transitive. Roughly speaking, we say that $i \sim_{r} i^{\prime}$ if $i^{\prime}$ is a "reversed" state of $i$. The following lemma shows that if two states $i$ and $j$ share a reversed state 
$i^{\prime}$ then the set of reversed states corresponding to $i$ and $j$ are the same.

Lemma 1. Consider an ergodic CTMC with state space $S$ and infinitesimal generator $\mathbf{Q}$. For all $i, j, i^{\prime} \in S$ such that $i \sim_{r} i^{\prime}$ and $j \sim_{r} i^{\prime}$, it holds that

$$
\left\{i^{\prime}: i \sim_{r} i^{\prime}\right\}=\left\{i^{\prime}: j \sim_{r} i^{\prime}\right\} .
$$

Proof: Let $i^{\prime \prime} \in S$ such that $i \sim_{r} i^{\prime \prime}$. We prove that also $j \sim_{r} i^{\prime \prime}$. From the facts that $i \sim_{r} i^{\prime}, j \sim_{r} i^{\prime}$ and $i \sim_{r} i^{\prime \prime}$ we have $q_{i}=q_{i}^{\prime}=q_{j}=q_{i^{\prime \prime}}$ and then Condition 1 of Definition 3 is satisfied. In order to prove Condition 2 of Definition 3, consider a finite sequence of one-step transitions $j=j_{1} \rightarrow$ $j_{2} \rightarrow \ldots \rightarrow j_{n-1} \rightarrow j_{n}$. From $j \sim_{r} i^{\prime}$ there exist $i_{n}^{\prime} \rightarrow$ $i_{n-1}^{\prime} \rightarrow \cdots \rightarrow i_{2}^{\prime} \rightarrow i_{1}^{\prime}=i^{\prime}$ such that $\left(j_{k}, i_{k}^{\prime}\right) \in \mathcal{R}$ for all $k \in\{1, \ldots, n\}$ and

$$
q_{j_{1} j_{2}} q_{j_{2} j_{3}} \cdots q_{j_{n-1} j_{n}} q_{j_{n} j_{1}}=q_{i_{1}^{\prime} i_{n}^{\prime}} q_{i_{n}^{\prime} i_{n-1}^{\prime}} \cdots q_{i_{3}^{\prime} i_{2}^{\prime}} q_{i_{2}^{\prime} i_{1}^{\prime}} .
$$

From $i \sim_{r} i^{\prime}$ there exist $i=i_{1} \rightarrow i_{2} \rightarrow \ldots \rightarrow i_{n-1} \rightarrow i_{n}$ such that $\left(i_{k}, i_{k}^{\prime}\right) \in \mathcal{R}$ for all $k \in\{1, \ldots, n\}$ and

$$
q_{i_{1} i_{2}} q_{i_{2} i_{3}} \cdots q_{i_{n-1} i_{n}} q_{i_{n} i_{1}}=q_{i_{1}^{\prime} i_{n}^{\prime}} q_{i_{n}^{\prime} i_{n-1}^{\prime}} \cdots q_{i_{3}^{\prime} i_{2}^{\prime}} q_{i_{2}^{\prime} i_{1}^{\prime}} .
$$

Finally, from $i \sim_{r} i^{\prime \prime}$ there exist $i_{n}^{\prime \prime} \rightarrow i_{n-1}^{\prime \prime} \rightarrow \cdots \rightarrow i_{2}^{\prime \prime} \rightarrow$ $i_{1}^{\prime \prime}=i^{\prime \prime} \in S$ such that $\left(i_{k}, i_{k}^{\prime \prime}\right) \in \mathcal{R}$ for all $k \in\{1, \ldots, n\}$ and

$$
q_{i_{1} i_{2}} q_{i_{2} i_{3}} \cdots q_{i_{n-1} i_{n}} q_{i_{n} i_{1}}=q_{i_{1}^{\prime \prime} i_{n}^{\prime \prime}} q_{i_{n}^{\prime \prime} i_{n-1}^{\prime \prime}} \cdots q_{i_{3}^{\prime \prime} i_{2}^{\prime \prime}} q_{i_{2}^{\prime \prime} i_{1}^{\prime \prime}}
$$

and hence

$$
q_{j_{1} j_{2}} q_{j_{2} j_{3}} \cdots q_{j_{n-1} j_{n}} q_{j_{n} j_{1}}=q_{i_{1}^{\prime \prime} i_{n}^{\prime \prime}} q_{i_{n}^{\prime \prime} i_{n-1}^{\prime \prime}} \cdots q_{i_{3}^{\prime \prime} i_{2}^{\prime \prime}} q_{i_{2}^{\prime \prime} i_{1}^{\prime \prime}} .
$$

Analogously, we can prove that for every sequence $i^{\prime \prime}=i_{1}^{\prime \prime} \rightarrow$ $i_{2}^{\prime \prime} \rightarrow \ldots \rightarrow i_{n-1}^{\prime \prime} \rightarrow i_{n}^{\prime \prime}$ there exist $j_{n} \rightarrow j_{n-1} \rightarrow \cdots \rightarrow j_{2} \rightarrow$ $j_{1}=j$ such that $\left(i_{k}^{\prime \prime}, j_{k}\right) \in \mathcal{R}$ for all $k \in\{1, \ldots, n\}$ and

$$
q_{i_{1}^{\prime \prime} i_{2}^{\prime \prime}} q_{i_{2}^{\prime \prime} i_{3}^{\prime \prime}} \cdots q_{i_{n-1}^{\prime \prime} i_{n}^{\prime \prime}} q_{i_{n}^{\prime \prime} i_{1}^{\prime \prime}}=q_{j_{1} j_{n}} q_{j_{n} j_{n-1}} \cdots q_{j_{3} j_{2}} q_{j_{2} j_{1}} .
$$

This concludes the proof that $j \sim_{r} i^{\prime \prime}$.

Reversal bisimilarity induces an equivalence relation over the states of the CTMC, denoted $\sim$, which equates states corresponding to the same set of reversed states. We call this equivalence relation, reversal equivalence.

Definition 5 (Reversal equivalence). Consider an ergodic CTMC with state space $S$ and reversal bisimilarity $\sim_{r} \subseteq S \times S$. We call reversal equivalence, denoted by $\sim$, the relation over $S$ defined as: for all $i, j \in S$,

$$
i \sim j \quad \text { iff } \quad\left\{i^{\prime}: i \sim_{r} i^{\prime}\right\}=\left\{i^{\prime}: j \sim_{r} i^{\prime}\right\} .
$$

Proposition 9. The relation defined by Definition 5 is an equivalence relation.

Proof: The proof trivially follows by Definition 5 .

The reversal equivalence $\sim \subseteq S \times S$ induces a partition on the state space $S$. Let $S / \sim$ denote the set of equivalences classes generated in this way. Let $[i] \in S / \sim$ denote the equivalence class containing $i \in S$, that is $[i]=\{j \in S \mid i \sim j\}$ and $n_{i}$ denote the cardinality of this set, that is $n_{i}=|[i]|=$ $|\{j \in S \mid i \sim j\}|$.
We are now ready to introduce our notion of autoreversibility for a given CTMC. The following definition states that a CTMC is autoreversible if it admits a reversal bisimilarity over its states which induces a weak similarity. A further condition relating forward and reverse transitions is required.

Definition 6 (Autoreversibility). An ergodic CTMC with state space $S$ and infinitesimal generator $\mathbf{Q}$ is said autoreversible if

1) $\sim_{r} \subseteq S \times S$ is a reversal bisimilarity over $S$,

2) $\sim \subseteq S \times S$ is a weak similarity,

3) for every $i, i^{\prime}, j, j^{\prime}$ such that $i \sim_{r} i^{\prime}$ and $j \sim_{r} j^{\prime}$,

$$
m_{i[j]}=m_{j^{\prime}\left[i^{\prime}\right]} .
$$

Example 2 (Autoreversibility). Consider the CTMC depicted by Figure 1. Since it has a finite number of states and is irreducible it is trivially ergodic. Assume $\lambda=2 a, \mu=a$, $\nu=8 a$ and $\rho=4 a$, with $a \in \mathbb{R}^{+}$, then it can be proved that the chain is autoreversible and the reversal bisimilarity $\sim_{r}$ is:

$\{(A, E),(C, E),(B, D),(F, B),(E, A),(E, C),(D, B),(B, F)\}$.

Then, the equivalence classes of $S / \sim$ are: $\{A, C\},\{D, F\}$, $\{B\},\{E\}$ with $n_{A}=n_{C}=2, n_{D}=n_{F}=2, n_{B}=1$ and $n_{E}=1$. Observe that $\{B\}$ is the class of the "reversed" of $\{A, C\}$ and $\{D, F\}$ is the class of the "reversed" of $\{E\}$.

The following proposition shows that $\sim_{r}$ is well-defined.

Proposition 10. Consider an ergodic CTMC with state space $S$, infinitesimal generator $\mathbf{Q}$ and reversal bisimilarity $\sim_{r} \subseteq$ $S \times S$. For every finite sequence of one-step transitions $i_{1} \rightarrow$ $i_{2} \rightarrow \ldots \rightarrow i_{n-1} \rightarrow i_{n}$ and $i_{n}^{\prime} \rightarrow i_{n-1}^{\prime} \rightarrow \cdots \rightarrow i_{2}^{\prime} \rightarrow i_{1}^{\prime}$ such that $i_{k} \sim_{r} i_{k}^{\prime}$ for all $k \in\{1, \ldots, n\}$ it holds that

$$
q_{i_{1} i_{2}} q_{i_{2} i_{3}} \cdots q_{i_{n-1} i_{n}} q_{i_{n} i_{1}}=q_{i_{1}^{\prime} i_{n}^{\prime}} q_{i_{n}^{\prime} i_{n-1}^{\prime}} \cdots q_{i_{3}^{\prime} i_{2}^{\prime}} q_{i_{2}^{\prime} i_{1}^{\prime}} .
$$

Proof: Let $i_{1}, i_{2}, \ldots, i_{n-1}, i_{n} \in S$ and $i_{1}^{\prime}=i_{1}^{\prime \prime} \in S$ be a state such that $i_{1} \sim_{r} i_{1}^{\prime \prime}$. By Definition 3, there exist $i_{n}^{\prime \prime}, i_{n-1}^{\prime \prime}, \ldots, i_{2}^{\prime \prime}, i_{1}^{\prime \prime} \in S$ such that $\left(i_{k}, i_{k}^{\prime \prime}\right) \in \mathcal{R}$ for all $k \in$ $\{1, \ldots, n\}$ and

$$
q_{i_{1} i_{2}} q_{i_{2} i_{3}} \cdots q_{i_{n-1} i_{n}} q_{i_{n} i_{1}}=q_{i_{1}^{\prime \prime} i_{n}^{\prime \prime}} q_{i_{n}^{\prime \prime} i_{n-1}^{\prime \prime}} \cdots q_{i_{3}^{\prime \prime} i_{2}^{\prime}} q_{i_{2}^{\prime \prime} i_{1}^{\prime \prime}} .
$$

By Lemma 1 and Definition 5, $i_{k}^{\prime} \sim i_{k}^{\prime \prime}$ for all $k \in\{1, \ldots, n\}$. Since $\sim$ is a weak similarity, the proof follows by Condition 1 of Definition 2.

We now show that $\sim_{r}$ is reflexive for the class of reversible Markov processes.

Proposition 11. For a stationary Markov process $X(t)$ with state space $S$, if $X(t)$ is reversible then $\sim_{r} \in S \times S$ is reflexive.

Proof: Let $X(t)$ be reversible. Then, by Proposition 1, for any finite sequence of states $i_{1}, i_{2}, \ldots, i_{n-1}, i_{n} \in S$, $q_{i_{1} i_{2}} q_{i_{2} i_{3}} \cdots q_{i_{n-1} i_{n}} q_{i_{n} i_{1}}=q_{i_{1} i_{n}} q_{i_{n} i_{n-1}} \cdots q_{i_{3} i_{2}} q_{i_{2} i_{1}}$. Consider $\mathcal{R}=\{(i, i): i \in S\}$. It is easy to see that $\mathcal{R}$ is a reversal bisimulation and hence $\mathcal{R} \subseteq \sim_{r}$, i.e., $\sim_{r}$ is reflexive.

In the following example we show Proposition 11 applied to the well-known reversible process called Birth\&Death process 


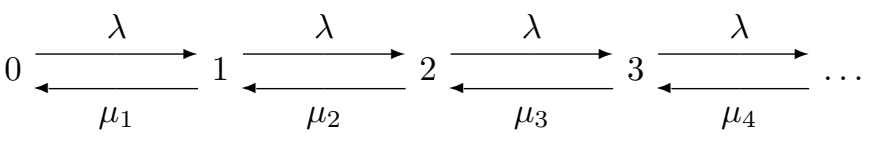

Fig. 2: Autoreversible Birth\&Death process.

which is underlying to the $\mathrm{M} / \mathrm{M} / \mathrm{n}$ queues with $n \in \mathbb{N} \backslash\{0\}$ or $n=\infty$.

Example 3 (Birth\&Death processes). Consider the Birth\&Death process of Figure 2. The CTMC is autoreversible and its reversal relation is reflexive since, given an arbitrary state $i$, each cycle of states starting from $i$ can be followed backward. Moreover, the sequence of states encountered by the forward and the backward paths are trivially associated by the reversal bisimulation.

Example 4 (Exponential queue with batch arrivals and departures). In this example we consider a Birth\&Death process with constant death rate, $\mu_{n}=\mu$ for all $n>0$ and constant arrival rate $\lambda$. We consider the possibility of bath arrivals of size $N$ and batch departures of the same size.

Let us denote by $\lambda_{N}$ and $\mu_{N}$ the batches' arrival and service rate. We assume that when there are less than $N$ customers in the queue the batch departure is disabled. We can easily prove that the CTMC underlying this model is autoreversible with a reflexive reverse relation if $\left(\lambda_{N} / \mu_{N}\right)=$ $(\lambda / \mu)^{N}$. Indeed, consider the path $n, n+1, \ldots, n+N$, then the product of the rates forming the forward cycle is $\lambda^{N} \mu_{N}$, while the product of the rates in the backward cycle is $\lambda_{N} \mu^{N}$. Since these two quantities must be identical we have the required condition dividing both hand-sides by $\mu_{N} \mu^{N}$. Notice that we can extend this analysis to batches of size $N_{1}, N_{2}, \ldots, N_{B}$ that arrive at and leave from the queue, obtaining the condition $(\lambda / \mu)^{N_{b}}=\lambda_{N_{b}} / \mu_{N_{b}}$ for all $b=1, \ldots, B$.

The following theorem plays an important role in the analysis of autoreversible CTMCs since it gives an effective way to compute their steady-state distribution without solving the system of global balance equations, i.e., by inspections of the transition rates.

Theorem 2 (Steady-state distribution). Consider an ergodic $C T M C$ with state space $S$, infinitesimal generator $\mathbf{Q}$, stationary probability $\pi$ and reversal bisimilarity $\sim_{r} \subseteq S \times S$. Let $i_{0} \in S$ be an arbitrary state. For all states $i \in S$, let $i=i_{n} \rightarrow i_{n-1} \rightarrow \cdots \rightarrow i_{1} \rightarrow i_{0}(n \geq 1)$ be a chain of one-step transitions and $i_{0}^{\prime} \rightarrow i_{1}^{\prime} \rightarrow \cdots \rightarrow i_{n-1}^{\prime} \rightarrow i_{n}^{\prime}=i^{\prime}$ such that $i_{k} \sim_{r} i_{k}^{\prime}$ for all $k \in\{0, \ldots, n\}$. Then

$$
\pi_{i}=C_{i_{0}} \frac{n_{i_{0}}}{n_{i}} \prod_{k=1}^{n} \frac{q_{i_{k-1}^{\prime} i_{k}^{\prime}}}{q_{i_{k} i_{k-1}}}
$$

where $C_{i_{0}} \in \mathbb{R}^{+}$.

Proof: First, we prove that Equation (6) gives a unique definition of $\pi_{i}$. Then, we will prove that it is the steady-state probability of state $i$ as required.
Observe that for all $j, k \in S$, we can find a chain $j \rightarrow$ $j_{1} \rightarrow \cdots \rightarrow j_{n-1} \rightarrow k$ (for $n \geq 1$ ) of one-step transitions since the Markov process is irreducible. Now we show that $\pi_{i}$ is well-defined. Indeed, if $i=j_{m} \rightarrow j_{m-1} \rightarrow \cdots \rightarrow$ $j_{1} \rightarrow j_{0}=i_{0}(m \geq 1)$ is another chain, we can always find a chain $i_{0}=h_{0} \rightarrow h_{1} \rightarrow \cdots \rightarrow h_{l-1} \rightarrow h_{l}=i$. Since the Markov process is autoreversible, there exist a chain $i^{\prime}=h_{l}^{\prime} \rightarrow h_{l-1}^{\prime} \rightarrow \cdots h_{1}^{\prime} \rightarrow h_{0}^{\prime}=i_{0}^{\prime \prime}=j_{0}^{\prime} \rightarrow j_{1}^{\prime} \rightarrow \cdots \rightarrow$ $j_{m-1}^{\prime} \rightarrow j_{m}^{\prime}=i^{\prime}$ such that $h_{k} \sim_{r} h_{k}^{\prime}$ for all $k \in\{0, \ldots, l\}$ and $j_{k} \sim_{r} j_{k}^{\prime}$ for all $k \in\{0, \ldots, m\}$, and

$$
\prod_{k=1}^{m} q_{j_{k} j_{k-1}} \prod_{k=1}^{l} q_{h_{k-1} h_{k}}=\prod_{k=1}^{l} q_{h_{k}^{\prime} h_{k-1}^{\prime}} \prod_{k=1}^{m} q_{j_{k-1}^{\prime} j_{k}^{\prime}} .
$$

Moreover, considering the one-step chain $i=i_{n} \rightarrow i_{n-1} \rightarrow$ $\cdots \rightarrow i_{1} \rightarrow i_{0}=h_{0} \rightarrow h_{1} \rightarrow \cdots \rightarrow h_{l-1} \rightarrow h_{l}=i$, by Definition 6 there exists a chain $i^{\prime \prime}=h_{l}^{\prime \prime} \rightarrow h_{l-1}^{\prime \prime} \rightarrow \cdots h_{1}^{\prime \prime} \rightarrow$ $h_{0}^{\prime \prime}=i_{0}^{\prime \prime} \rightarrow i_{1}^{\prime \prime} \rightarrow \cdots \rightarrow i_{n-1}^{\prime \prime} \rightarrow i_{n}^{\prime \prime}$ such that $h_{k} \sim_{r} h_{k}^{\prime \prime}$ for all $k \in\{0, \ldots, l\}, i_{k} \sim_{r} i_{k}^{\prime \prime}$ for all $k \in\{0, \ldots, n\}$, and

$$
\prod_{k=1}^{n} q_{i_{k} i_{k-1}} \prod_{k=1}^{l} q_{h_{k-1} h_{k}}=\prod_{k=1}^{l} q_{h_{k}^{\prime \prime} h_{k-1}^{\prime \prime}} \prod_{k=1}^{n} q_{i_{k-1}^{\prime \prime} i_{k}^{\prime \prime} .}
$$

By Proposition 10, $h_{k}^{\prime} \sim h_{k}^{\prime \prime}$ for all $k \in\{0, \ldots, l\}$ and also $i_{k}^{\prime} \sim_{r} i_{k}^{\prime \prime}$ for all $k \in\{0, \ldots, n\}$. Hence by Condition 1 of Definition 2,

$$
\prod_{k=1}^{l} q_{h_{k}^{\prime \prime} h_{k-1}^{\prime \prime}} \prod_{k=1}^{n} q_{i_{k-1}^{\prime \prime} i_{k}^{\prime \prime}}=\prod_{k=1}^{l} q_{h_{k}^{\prime} h_{k-1}^{\prime}} \prod_{k=1}^{n} q_{i_{k-1}^{\prime} i_{k}^{\prime}}
$$

and then

$$
\prod_{k=1}^{n} q_{i_{k} i_{k-1}} \prod_{k=1}^{l} q_{h_{k-1} h_{k}}=\prod_{k=1}^{l} q_{h_{k}^{\prime} h_{k-1}^{\prime}} \prod_{k=1}^{n} q_{i_{k-1}^{\prime} i_{k}^{\prime}} .
$$

From Equations (7) and (8), we obtain

$$
\prod_{k=1}^{m} \frac{q_{j_{k-1}^{\prime} j_{k}^{\prime}}}{q_{j_{k} j_{k-1}}}=\prod_{k=1}^{n} \frac{q_{i_{k-1}^{\prime} i_{k}^{\prime}}}{q_{i_{k} i_{k-1}}} .
$$

Hence

$$
\pi_{i}=C_{i_{0}} \frac{n_{i_{0}}}{n_{i}} \prod_{k=1}^{n} \frac{q_{i_{k-1}^{\prime} i_{k}^{\prime}}}{q_{i_{k} i_{k-1}}}
$$

where $C_{i_{0}}$ is a positive constant, is well-defined.

In order to prove that Equation (6) is the stationary probability of state $i$, we use the approach described in Section II. Since the CTMC is stationary, we can define its reversed whose transition matrix $\mathbf{Q}^{R}$ is defined according to Lemma 2 . Let us assume Equation (6) and we show that the reversed process satisfies the generalised Kolmogorov's criteria of Proposition 3. By uniqueness of the steady-state distribution we will conclude the proof.

Let us consider an arbitrary transition from state $i$ to $j$ with rate $q_{i j}$ in the forward chain, then the corresponding transition in the reversed process goes from $j$ to $i$ with rate $q_{j i}^{R}$. Observe that we have just proved that we can choose an arbitrary path from $i$ to the reference state $i_{0}$, in particular we can choose the path going from $i$ to $j$ and then a path from $j$ to $i_{0}$. By 
Definition 6 there will surely exist $i_{0}^{\prime}, i^{\prime}, j^{\prime}$ such that $i_{0} \sim_{r} i_{0}^{\prime}$, $i \sim_{r} i^{\prime}$ and $j \sim_{r} j^{\prime}$ and a path going from $i_{0}^{\prime}$ to $j^{\prime}$ and one step from $j^{\prime}$ to $i^{\prime}$ such that

$$
\pi_{i}=\frac{\Psi_{i_{0} \rightarrow j}^{\prime} q_{j^{\prime} i^{\prime}}}{\Psi_{j \rightarrow i_{0}} q_{i j}} \quad \pi_{j}=\frac{\Psi_{i_{0} \rightarrow j}^{\prime}}{\Psi_{j \rightarrow i_{0}}}
$$

where $\Psi_{i_{0} \rightarrow j}^{\prime}=\prod_{k=1}^{n} q_{i_{k-1}^{\prime} i_{k}^{\prime}}$ and $\Psi_{j \rightarrow i_{0}}=\prod_{k=1}^{n} q_{i_{k} i_{k-1}}$ with $j=i_{n}$.

By Equation (2), we have:

$$
q_{j i}^{R}=\frac{\pi_{i}}{\pi_{j}} q_{i j}=\frac{C_{i_{0}} \frac{n_{i_{0}}}{n_{i}} \frac{\Psi_{i_{0} \rightarrow j}^{\prime} q_{j^{\prime} i^{\prime}}}{\Psi_{j \rightarrow i_{0}} q_{i j}}}{C_{i_{0}} \frac{n_{i_{0}}}{n_{j}} \frac{\Psi_{i_{0} \rightarrow j}^{\prime}}{\Psi_{j \rightarrow i_{0}}}} q_{i j}=\frac{n_{j}}{n_{i}} q_{j^{\prime} i^{\prime}} .
$$

The generalised Kolmogorov's criteria on the cycles is readily verified. Consider the sequence of states $i_{1}, \ldots, i_{n}$ associated with the product $q_{i_{1} i_{2}} \cdots q_{i_{n-1} i_{n}} q_{i_{n} i_{1}}$, then the product of the rates in the reversed process is:

$$
q_{i_{1} i_{n}}^{R} q_{i_{n} i_{n-1}}^{R} \cdots q_{i_{2} i_{1}}^{R}=\frac{n_{i_{1}}}{n_{i_{n}}} q_{i_{1}^{\prime} i_{n}^{\prime}} \frac{n_{i_{n}}}{n_{i_{n-1}}} q_{i_{n}^{\prime} i_{n-1}^{\prime}} \cdots \frac{n_{i_{2}}}{n_{i_{1}}} q_{i_{2}^{\prime} i_{1}^{\prime}} .
$$

After simplifying we obtain an identity by Definition 6 .

We now verify the first generalised Kolmogorov's criteria. Let us consider an arbitrary state $j$, then the outgoing flow from the reversed process is:

$$
q_{j}^{R}=\sum_{\substack{i \in S \\ q_{j i}^{R}>0}} q_{j i}^{R}=\sum_{\substack{i \in S \\ q_{i j}>0}} \frac{n_{j}}{n_{i}} q_{j^{\prime} i^{\prime}}
$$

We prove that

$$
q_{j^{\prime}}=\sum_{\substack{i \in S \\ q_{i j}>0}} \frac{n_{j}}{n_{i}} q_{j^{\prime} i^{\prime}}
$$

and this will conclude the proof since, by Definition $3, q_{j}^{\prime}=q_{j}$. Let us consider the right-hand-side of Equation (9), then we have:

$$
\begin{aligned}
& \sum_{\substack{i \in S \\
q_{i j}>0}} \frac{n_{j}}{n_{i}} q_{j^{\prime} i^{\prime}}=\sum_{[i] \in S / \sim} \sum_{\substack{i \in[i] \\
q_{i j}>0}} \frac{n_{j}}{n_{i}} q_{j^{\prime} i^{\prime}} \\
& =\sum_{[i] \in S / \sim} m_{[i] j} \frac{n_{j}}{n_{i}} q_{j^{\prime} i^{\prime}}=\sum_{[i] \in S / \sim} \frac{m_{i[j]} n_{i}}{n_{i}} q_{j^{\prime} i^{\prime}},
\end{aligned}
$$

where the last equality follows from Proposition 7. By Condition 3 of Definition 6 we have $m_{i[j]}=m_{j^{\prime}\left[i^{\prime}\right]}$ and since every equivalence class has exactly one counterpart (possibly itself) by definition, we conclude the proof:

$$
\sum_{[i] \in S / \sim} m_{j^{\prime}\left[i^{\prime}\right]} q_{j^{\prime} i^{\prime}}=\sum_{\left[i^{\prime}\right] \in S / \sim} m_{j^{\prime}\left[i^{\prime}\right]} q_{j^{\prime} i^{\prime}}=\sum_{i^{\prime} \in S / \sim} q_{j^{\prime} i^{\prime}}=q_{j}^{\prime} .
$$

We now state two corollaries that aim at simplifying the application of Theorem 2 .

Corollary 1. Consider an ergodic CTMC with state space $S$, infinitesimal generator $\mathbf{Q}$, stationary probability $\pi$ and reversal bisimilarity $\sim_{r} \subseteq S \times S$. Then for all $i, j \in S$ such that $i=i_{n} \rightarrow i_{n-1} \rightarrow \cdots \rightarrow i_{1} \rightarrow i_{0}=j$ and $j^{\prime}=i_{0}^{\prime} \rightarrow i_{1}^{\prime} \rightarrow \cdots \rightarrow i_{n-1}^{\prime} \rightarrow i_{n}^{\prime}=i^{\prime}$ with $i_{k} \sim_{r} i_{k}^{\prime}$ for all $k \in\{0, \ldots, n\}$, it holds

$$
n_{i} \pi_{i}=n_{j} \pi_{j} \prod_{k=1}^{n} \frac{q_{i_{k-1}^{\prime} i_{k}^{\prime}}}{q_{i_{k} i_{k-1}}} .
$$

Proof: By Theorem 2,

$$
\pi_{i}=C_{j} \frac{n_{j}}{n_{i}} \prod_{k=1}^{n} \frac{q_{i_{k-1}^{\prime} i_{k}^{\prime}}}{q_{i_{k} i_{k-1}}}
$$

where $C_{j}$ is a positive constant. In particular, $\pi_{j}=C_{j}$ and hence

$$
\pi_{i}=\pi_{j} \frac{n_{j}}{n_{i}} \prod_{k=1}^{n} \frac{q_{i_{k-1}^{\prime} i_{k}^{\prime}}}{q_{i_{k} i_{k-1}}}
$$

Corollary 2. Consider an ergodic CTMC with state space $S$, infinitesimal generator $\mathbf{Q}$, stationary probability $\boldsymbol{\pi}$ and reversal bisimilarity $\sim_{r} \subseteq S \times S$. Then for all $i, j \in S$ with $q_{j i}>0$ and for all $i^{\prime}, j^{\prime} \in S$ such that $i \sim_{r} i^{\prime}, j \sim_{r} j^{\prime}$ and $q_{i^{\prime} j^{\prime}}>0$ it holds

$$
n_{j} \pi_{j} q_{j i}=n_{i} \pi_{i} q_{i^{\prime} j^{\prime}}
$$

Proof: Let $i, j \in S$ such that $i \sim_{r} i^{\prime}, j \sim_{r} j^{\prime}$ and $q_{i^{\prime} j^{\prime}}>$ 0 . Let $i_{0} \in S$ be an arbitrary state, $i=i_{n} \rightarrow i_{n-1} \rightarrow \cdots \rightarrow$ $i_{1} \rightarrow i_{0}(n \geq 1)$ be a chain of one-step transitions and $i_{0}^{\prime} \rightarrow$ $i_{1}^{\prime} \rightarrow \cdots \rightarrow i_{n-1}^{\prime} \rightarrow i_{n}^{\prime}=i^{\prime}$ such that $i_{k} \sim_{r} i_{k}^{\prime}$ for all $k \in\{0, \ldots, n\}$. By Theorem 2,

$$
\pi_{i}=C_{i_{0}} \frac{n_{i_{0}}}{n_{i}} \prod_{k=1}^{n} \frac{q_{i_{k-1}^{\prime} i_{k}^{\prime}}}{q_{i_{k} i_{k-1}}}
$$

where $C_{i_{0}}$ is a positive constant. Now suppose that $q_{j i}>0$ and $q_{i^{\prime} j^{\prime}}>0$. Again, by Theorem 2,

$$
\pi_{j}=C_{i_{0}} \frac{n_{i_{0}}}{n_{j}} \frac{q_{i^{\prime} j^{\prime}}}{q_{j i}} \prod_{k=1}^{n} \frac{q_{i_{k-1}^{\prime} i_{k}^{\prime}}}{q_{i_{k} i_{k-1}}}
$$

and hence

$$
n_{j} \pi_{j} q_{j i}=n_{i} \pi_{i} q_{i^{\prime} j^{\prime}}
$$

We apply Theorem 2 to our running example.

Example 5. Let us consider again the CTMC depicted in Figure 1 with the reversal bisimilarity and the reversal equivalence derived in Example 2. Let us choose an arbitrary reference state $i_{0}=A$ and then pick the shortest sequence of states from any other state $i$ to $A$ (whose reversed is $E$ ). Notice that $n_{A}=2$ since it stays in an equivalence class of cardinality 2. For instance, take $B$ whose reversed is either $D$ or $F$ and $n_{B}=1$. Then we have:

$$
\pi_{B}=C_{A} \frac{2}{1} \frac{q_{E D}}{q_{B A}}=C_{A} .
$$


In a similar way we obtain:

$$
\begin{aligned}
& \pi_{C}=C_{A} \frac{2}{2} \frac{q_{E F} q_{F B} q_{B E}}{q_{C F} q_{F B} q_{B A}}=C_{A} \frac{\rho \mu \nu}{\lambda^{2} \nu}=C_{A} \\
& \pi_{D}=C_{A} \frac{2}{2} \frac{q_{E D} q_{D B}}{q_{D B} q_{B A}}=C_{A} \frac{\nu \mu}{\lambda \nu}=\frac{C_{A}}{2} \\
& \pi_{E}=C_{A} \frac{2}{1} \frac{q_{E F} q_{F B} q_{B C}}{q_{E F} q_{F B} q_{B A}}=2 C_{A} \frac{\lambda}{\lambda}=2 C_{A} \\
& \pi_{F}=C_{A} \frac{2}{2} \frac{q_{E D} q_{D B}}{q_{F B} q_{B A}}=C_{A} \frac{\mu \nu}{\nu \lambda}=\frac{C_{A}}{2}
\end{aligned}
$$

We can now derive $\pi_{A}=C_{A}$ and by imposing $\sum_{i \in S} \pi_{A}=1$ this gives $C_{A}=1 / 6$.

The next Theorem shows that equivalent states have the same stationary probability.

Theorem 3. Consider an ergodic CTMC with state space $S$, infinitesimal generator $\mathbf{Q}$, stationary probability $\pi$ and reversal equivalence $\sim \subseteq S \times S$. For all states $i, j \in S$ such that $i \sim j$ it holds $\pi_{i}=\pi_{j}$.

Proof: Since the Markov chain satisfies the conditions of Definition 2 by hypothesis, then the result follows by Theorem 1.

The following theorem establishes the relation between the stationary probability of a state $i$ and that of its reversed $i^{\prime}$. We will show that this relation highly improves the efficiency of the computation of the steady-state distribution for autoreversible processes.

Theorem 4. Consider an ergodic CTMC with state space $S$, infinitesimal generator $\mathbf{Q}$, stationary probability $\boldsymbol{\pi}$ and reversal bisimilarity $\sim_{r} \subseteq S \times S$. For all states $i, i^{\prime} \in S$ such that $i \sim_{r} i^{\prime}$ it holds $n_{i} \pi_{i}=n_{i^{\prime}} \pi_{i^{\prime}}$.

Proof: Consider the following chain of one step transitions: $i=i_{n} \rightarrow i_{n-1} \rightarrow \cdots \rightarrow i_{0}=i^{\prime}$. Let $i_{0}^{\prime} \rightarrow \cdots \rightarrow$ $i_{n-1}^{\prime} \rightarrow i_{n}^{\prime}$ such that $i_{k} \sim_{r} i_{k}^{\prime}$ for $k \in\{0, \ldots, n\}$. By Theorem 2,

$$
n_{i} \pi_{i}=n_{i^{\prime}} \pi_{i^{\prime}} \prod_{k=1}^{n} \frac{q_{i_{k-1}^{\prime}} i_{k}^{\prime}}{q_{i_{k} i_{k-1}}} .
$$

Let $i^{\prime}=j_{m} \rightarrow j_{m-1} \rightarrow \cdots \rightarrow j_{0}=i$ and $j_{0}^{\prime} \rightarrow \cdots \rightarrow$ $j_{m-1}^{\prime} \rightarrow j_{m}^{\prime}$ such that $j_{k} \sim_{r} j_{k}^{\prime}$ for $k \in\{0, \ldots, n\}$. By definition of autoreversibility,

$$
\prod_{k=1}^{m} q_{j_{k} j_{k-1}} \prod_{k=1}^{n} q_{i_{k-1}^{\prime} i_{k}^{\prime}}=\prod_{k=1}^{n} q_{i_{k} i_{k-1}} \prod_{k=1}^{m} q_{j_{k-1}^{\prime} j_{k}^{\prime}}
$$

and also

$$
\prod_{k=1}^{m} q_{j_{k} j_{k-1}} \prod_{k=1}^{n} q_{i_{k} i_{k-1}}=\prod_{k=1}^{n} q_{i_{k-1}^{\prime} i_{k}^{\prime}} \prod_{k=1}^{m} q_{j_{k-1}^{\prime} j_{k}^{\prime}} .
$$

By equations (10) and (11),

$$
\prod_{k=1}^{n} \frac{q_{i_{k-1}^{\prime} i_{k}^{\prime}}}{q_{i_{k} i_{k-1}}}=\prod_{k=1}^{m} \frac{q_{j_{k-1}^{\prime} j_{k}^{\prime}}}{q_{j_{k} j_{k-1}}}=\prod_{k=1}^{m} \frac{q_{j_{k} j_{k-1}}}{q_{j_{k-1}^{\prime} j_{k}^{\prime}}}
$$

and hence

$$
\prod_{k=1}^{n} \frac{q_{i_{k-1}^{\prime} i_{k}^{\prime}}}{q_{i_{k} i_{k-1}}}=1
$$

which proves $n_{i} \pi_{i}=n_{i^{\prime}} \pi_{i^{\prime}}$.

We give an example of application of Theorems 3 and 4 .

Example 6. Let us reconsider the steady-state probabilities derived in Example 5. Notice that from Theorems 3 and 4 we immediately know that given $\pi_{A}=C_{A}$ we have $\pi_{C}=$ $C_{A}$ because they belong to the same equivalence class, and also $\pi_{E}=2 C_{A}$ since $E$ is the inverse of $A$ (and $C$ ) but its equivalence class has cardinality 1 . Then we can compute $\pi_{E}$ as done in Example 5 and using again Theorem 3 we immediately derive the remaining stationary probabilities. In practice, Theorem 3 reduces the number of cycles one has to consider to compute the process' stationary distribution. Specifically, in this example we have to consider only one cycle.

\section{EXAMPLES}

In this section we illustrate some examples of autoreversible processes. Clearly, all the product-form models that are reversible are also autoreversible (see, e.g., [16], [21], [22]) in which a reflexive reversal bisimilarity can be defined. For this reason we will focus on non-product-form models and show that the notion of autoreversibility simplifies the computation of the stationary distribution.

Moreover, it is worth notice that the CTMCs with the regular structures that are required by autoreversibility often underlie Markovian process algebra cooperations. For the sake of readability we briefly introduce a Markovian process algebra, i.e., the Performance Evaluation Process Algebra (PEPA) [3]. We consider a reduced syntax of PEPA. Prefix: $(a, \lambda) . P$ is the agent that performs an activity of type $a$ whose duration is an exponentially distributed random variable with parameter $\lambda$ and then behaves as $P$. Symbol $T$ denotes that the duration of an activity is determined by another agent. Choice: The choice operator $P+Q$ describes an agent that can choose to behave as $P$ or $Q$ according to the standard race policy [3] (i.e., the fastest sampled time determines the activity to carry out). Constant: A new constant agent $A$ is defined to behave as $P$ by writing $A \stackrel{\text { def }}{=} P$. Cooperation: The modularity of this Markovian process algebra strongly depends on the operator specifying the cooperation among two agents: $P \triangle Q$. In this case, all the transitions in $P$ and $Q$ whose type belongs to the set $L$ can be carried out only jointly. The rate of the joint transition must be decided according to the rules described in the semantics [3]. In particular, in case of cooperation on a type $a$ between an activity with a specified rate $\lambda$ (active) and one with unspecified rate $T$, the joint activity has type $a$ and rate $\lambda$.

Example 7. This example aims at showing the simplest example of a non-product-form cooperation between two agents that is autoreversible. Let us consider the following PEPA components: 


$$
\begin{aligned}
& P_{1} \stackrel{\text { def }}{=}(a, \alpha) \cdot P_{2} \quad Q_{1} \stackrel{\text { def }}{=}(a, \top) \cdot Q_{2} \\
& P_{2} \stackrel{\text { def }}{=} \quad(b, \beta) . P_{1} \quad Q_{2} \stackrel{\text { def }}{=} \quad(c, \gamma) \cdot Q_{1} .
\end{aligned}
$$

Now consider the system Sys $s_{1}$ defined by: Sys $s_{1} \stackrel{\text { def }}{=} P_{1} \underset{\{a\}}{\bigotimes} Q_{1}$ whose derivation graph is:

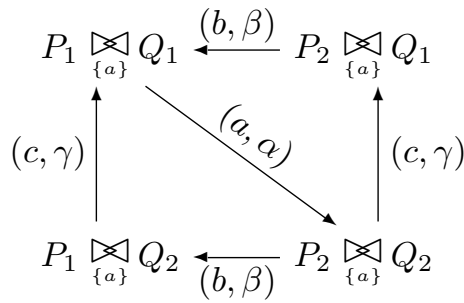

The underlying CTMC has the same state space $S$ and is:

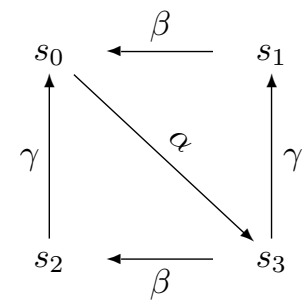

with $s_{0}=P_{1} \underset{\{a\}}{\bowtie} Q_{1}, s_{1}=P_{2} \underset{\{a\}}{\bowtie} Q_{1}, s_{2}=P_{1} \underset{\{a\}}{\bowtie} Q_{2}$ and $s_{3}=P_{2} \underset{\{a\}}{\bigotimes} Q_{2}$. Consider the relation

$$
\mathcal{R}=\left\{\left(s_{0}, s_{3}\right),\left(s_{1}, s_{2}\right)\right\} .
$$

It is easy to see that if $\alpha=\beta+\gamma$ and $\beta=\gamma$ then $\mathcal{R}$ is a reversal bisimilarity over $\left\{s_{0}, s_{1}, s_{2}, s_{3}\right\}$, and the above CTMC is autoreversible (but not reversible). To derive the steady-state distribution: fix a state, e.g., $s_{0}$ with $\pi_{s_{0}}=C>0$. It immediately follows that its inverse has the same stationary probability, i.e., $\pi_{s_{3}}=\pi_{s_{0}}$. The computation of $\pi_{s_{1}}$ follows by Theorem 2 considering the path from the inverse of $s_{0}$, i.e., $s_{3}$ to the inverse of $s_{1}$, i.e., $s_{2}$ and dividing its rate by the transition rate of the forward path from $s_{1}$ to $s_{0}$. This gives $\pi_{s_{1}}=\pi_{s_{0}}$ and hence also $\pi_{s_{2}}=\pi_{s_{0}}$.

Example 8. Consider the following PEPA components:

$$
\begin{array}{lll}
P_{1} & \stackrel{\text { def }}{=} & (a, \alpha) \cdot P_{2}+(e, \alpha) \cdot P_{2} \\
P_{2} & \stackrel{\text { def }}{=} & (b, \beta) \cdot P_{1} \\
& \\
Q_{1} & \stackrel{\text { def }}{=} & (a, \top) \cdot Q_{2} \\
Q_{2} & \stackrel{\text { def }}{=} & (c, \gamma) \cdot Q_{1}+(d, \delta) \cdot Q_{3} \\
Q_{3} & \stackrel{\text { def }}{=} & (e, \top) \cdot Q_{2}
\end{array}
$$

and the system Sys 2 defined by:

$$
S y s_{2} \stackrel{\text { def }}{=} P_{1} \underset{\{a, e\}}{\bowtie} Q_{1} \text {. }
$$

The derivation graph of $\mathrm{Sys}_{2}$ is

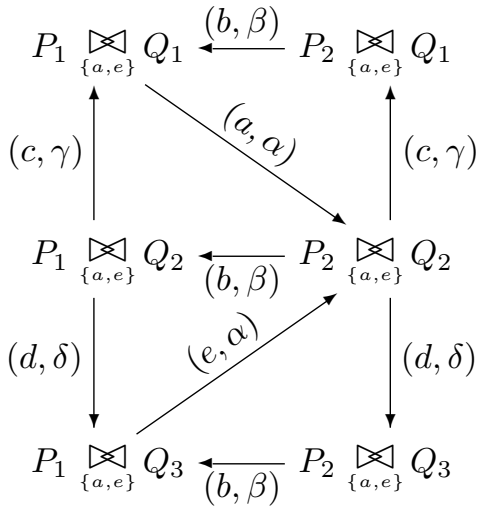

Notice that with the opportune rate conditions, the underlying CTMC is that of the running example of Figure 1.

Example 9. Consider the PEPA components depicted below:

$$
\begin{array}{lll}
P_{1} & \stackrel{\text { def }}{=} & \left(a, \alpha_{1}\right) \cdot P_{2}+\left(b, \alpha_{2}\right) \cdot P_{2} \\
P_{2} & \stackrel{\text { def }}{=} & (c, \beta) \cdot P_{1}+(d, \beta) \cdot P_{3} \\
P_{3} & \stackrel{\text { def }}{=} & (a, \top) \cdot P_{2}+(b, \top) \cdot P_{2} \\
Q_{1} & \stackrel{\text { def }}{=} & (a, \alpha) \cdot Q_{2} \\
Q_{2} & \stackrel{\text { def }}{=} & (e, \beta) \cdot Q_{1}+(f, \beta) \cdot Q_{3} \\
Q_{3} & \stackrel{\text { def }}{=} & (b, \alpha) \cdot Q_{2}
\end{array}
$$

with $\alpha_{1} \geq \alpha$ and $\alpha_{2} \geq \alpha$. Let Sys 3 defined by:

$$
S y s_{3} \stackrel{\text { def }}{=} P_{1} \underset{\{a, b\}}{\bowtie} Q_{1} \text {. }
$$

The derivation graph $\mathrm{Sys}_{3}$ is defined by:

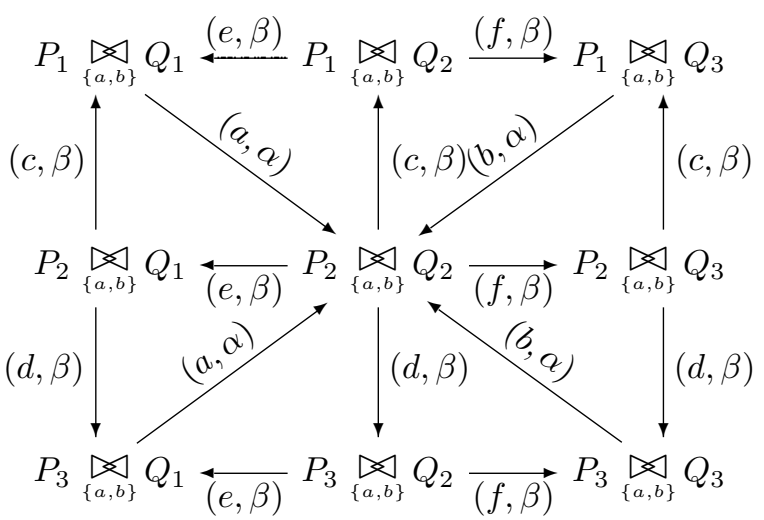

It is easy to see that its underlying CTMC is autoreversible.

Example 10. We consider a last example that aims at illustrating the methodology for the computation of the steadystate probabilities for autoreversible processes. We consider the following ergodic CTMC: 


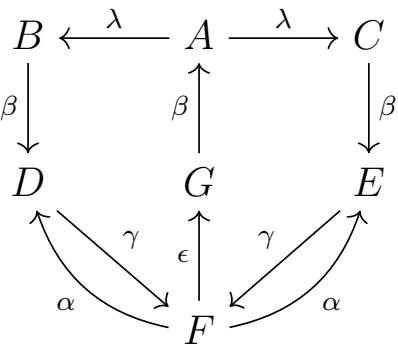

In this case we have the following equivalence classes: $\{C, B\}$ that contains the inverse states of $\{G\},\{D, E\}$ that contains the inverse states of $\{F\}$ and, finally, $\{A\}$ which is inverse of itself, provided that $2 \alpha+\epsilon=\gamma$. Let us compute the steady-state distribution starting from the reference state $B$, i.e., $\pi_{B}=\pi_{C}=K>0$. We immediately derive $\pi_{G}=2 K$. To compute $\pi_{A}$ we choose the path from $A$ to $C$ and its inverse $G \rightarrow A$, obtaining, by Theorem $2, \pi_{A}=\pi_{C} \beta / \lambda=K \beta / \lambda$. We can derive $\pi_{F}$ considering $F \rightarrow G$ and its inverse $C \rightarrow E$, obtaining $\pi_{F}=\pi_{G} \beta / \epsilon=2 K \beta / \epsilon$. Then we immediately derive the probability of its inverses $\pi_{D}=\pi_{E}=\pi_{F} / 2=K \beta / \epsilon$. The value of $K$ is obtained by normalising the probabilities.

\section{CONCLUSION}

In this paper we have characterised a class of CTMCs called autoreversible whose steady-state analysis is particularly efficient. The notion of autoreversibility is related to that of lumping [15] and similarity of states [17] as discussed in Section III where we introduce the definition of weak similarity. Weak similarity among states is different from Yap's definition of state similarity [17] and for reversible CTMCs we can say that weak similarity generalises the notion of similarity. Autoreversibility is introduced in Section IV and it is defined in terms of a symmetric coinductive relation, called reversal bisimilarity, which induces an equivalence relation among the states of a CTMC called reversal equivalence. We have shown that every reversible CTMC is also autoreversible: each equivalence class has a singleton and, roughly speaking, is the inverse of itself. However, we have shown examples of CTMCs that are autoreversible but not reversible (nor quasireversible). Finally, in Section V we showed that the regular structures required by autoreversibility are often generated by the operators for the cooperation in Markovian process algebras. It is worth notice that similarly to what happens when using product-form solutions, autoreversibility requires strong conditions in the structure of the CTMC [9] and often also on its transition rates [23], [24]. Nevertheless, the improvement in the computation of the steady-state distribution of autoreversible processes is very high and, differently for what happens for lumped models, it does not need the solution of the system of global balance equations.

Future research efforts will be addressed to study the implications of autoreversibility at a higher level of abstraction, for instance by characterising the class of Markovian process algebra agents originating autoreversible CTMCs.

\section{ACKNOWLEDGMENTS}

Work partially supported by the MIUR Project CINA: "Compositionality, Interaction, Negoziation, Autonomicity for the future ICT society".

\section{REFERENCES}

[1] R. J. Boucherie and N. M. van Dijk, Queueing Networks: A Fundamental Approach, ser. International Series in Operations Research \& Management Science. Springer, 2011.

[2] M. K. Molloy, "Performance analysis using stochastic Petri nets," IEEE Trans. on Comput., vol. 31, no. 9, pp. 913-917, 1982.

[3] J. Hillston, A Compositional Approach to Performance Modelling. Cambridge Press, 1996.

[4] N. Götz, U. Herzog, and M. Rettelbach, "Multiprocessor and distributed system design: The integration of functional specification and performance analysis using stochastic process algebras," in Performance Evaluation of Computer and Communication Systems, ser. Lecture Notes in Computer Science, 1993, vol. 729, pp. 121-146.

[5] B. Plateau, "On the stochastic structure of parallelism and synchronization models for distributed algorithms," SIGMETRICS Perf. Eval. Rev., vol. 13, no. 2, pp. 147-154, 1985.

[6] W. J. Stewart, Probability, Markov Chains, Queues, and Simulation. UK: Princeton University Press, 2009.

[7] _ Introduction to the Numerical Solution of Markov Chains. UK: Princeton University Press, 1994.

[8] E. Gelenbe, "Random neural networks with negative and positive signals and product form solution," Neural Computation, vol. 1, no. 4, pp. 502$510,1989$.

[9] P. G. Harrison, "Turning back time in Markovian process algebra," Theoretical Computer Science, vol. 290, no. 3, pp. 1947-1986, 2003.

[10] P. Harrison and J. Hillston, "Exploiting quasi-reversible structures in Markovian process algebra models," The Computer Journal, vol. 38, no. 7, pp. 510-520, 1995.

[11] P. G. Harrison, "Process algebraic non-product-forms," Electronic Notes Theor. Comput. Sci., vol. 151, no. 3, 2006.

[12] N. Thomas and P. Harrison, "State-dependent rates and semi-productform via the reversed process," in Proc. of the 7th European performance engineering conference on Computer performance engineering, ser. LNCS, vol. 6342. Springer-Verlag, 2010, pp. 207-218.

[13] A. Marin, S. Balsamo, and P. Harrison, "Analysis of stochastic Petri nets with signals," Perf. Eval., vol. 85, no. 7, pp. 1520-1539, 2012.

[14] M. F. Neuts, Matrix Geometric Solutions in Stochastic Models. Baltimore, Md: John Hopkins, 1981.

[15] J. G. Kemeny and J. L. Snell, Finite Markov Chains. D. Van Nostrand Company, inc., 1960, ch. II.

[16] F. Kelly, Reversibility and stochastic networks. New York: Wiley, 1979.

[17] V. Yap, "Similar states in continuous-time Markov chains," J. Appl. Prob., vol. 46, pp. 497-506, 2009.

[18] R. Milner, Communication and Concurrency. Prentice-Hall, 1989.

[19] R. Paige and R. E. Tarjan, "Three Partition Refinement Algorithms," SIAM Journal on Computing, vol. 16, no. 6, pp. 973-989, 1987.

[20] A. Dovier, C. Piazza, and A. Policriti, "An efficient algorithm for computing bisimulation equivalence," Theor. Comput. Sci., vol. 311, no. 1-3, pp. 221-256, 2004.

[21] S. Balsamo, P. G. Harrison, and A. Marin, "A unifying approach to product-forms in networks with finite capacity constraints," in Proc. of the 2010 ACM SIGMETRICS Int. Conf. on Measurement and Modeling of Computer Systems, V. Misra, P. Barford, and M. S. Squillante, Eds., New York, NY, USA, 14-18 June 2010, pp. 25-36.

[22] J. Hillston and N. Thomas, "A syntactical analysis of reversible pepa models," in Proc. of 6th Int. Workshop on Process Algebra and Performance Modelling, 1998, pp. 37-49.

[23] J. L. Coleman, W. Henderson, and P. G. Taylor, "Product form equilibrium distributions and a convolution algorithm for Stochastic Petri nets," Perf. Eval., vol. 26, no. 3, pp. 159-180, 1996.

[24] S. Balsamo, P. G. Harrison, and A. Marin, "Methodological Construction of Product-form Stochastic Petri-Nets for Performance Evaluation," $J$. of System and Software, vol. 85, no. 7, pp. 1520-1539, 2012. 